EGU21-4571

https://doi.org/10.5194/egusphere-egu21-4571

EGU General Assembly 2021

(c) Author(s) 2021. This work is distributed under

the Creative Commons Attribution 4.0 License.

\title{
From field to stream: Tracing streambed organic carbon origins at a catchment scale
}

Katy Wiltshire ${ }^{1}$, Miriam Glendell ${ }^{2}$, Toby Waine ${ }^{1}$, Robert Grabowski ${ }^{1}$, Barry Thornton ${ }^{2}$, and Jeroen Meersmans $^{3}$

${ }^{1}$ Cranfield University, School of Water, Energy and Environment, Bedford, United Kingdom

${ }^{2}$ The James Hutton Institute, Craigiebuckler, AB15 8QH, Aberdeen, Scotland, United Kingdom

${ }^{3}$ University of Liège, TERRA Teaching and Research Centre, Gembloux Agro-Bio Tech, Gembloux, 5030, Belgium

Quantifying organic carbon (OC) levels and the processes altering them is key in unlocking soils potential as a mediator of climate change through sequestration of atmospheric $\mathrm{CO}_{2}$. In areas of high soil erosion increased fluxes of $O C$ across the terrestrial-aquatic interface are likely and understanding these fluxes is crucial in integrating lateral OC fluxes within the carbon cycle. For this study of a small UK catchment, OC mapping and Revised Universal Soil Loss Equation (RUSLE) based erosion modelling provided estimates of proportional soil OC loss coming from each land use. Sediment fingerprinting using $n$-alkane biomarkers and a Bayesian unmixing model provided a comparison of streambed OC proportions by land use to assess which processes were dominating OC input to streams. Results showed that RUSLE-based soil OC loss proportions exhibited disconnect with sediment fingerprinting OC composition and the river corridor and riparian environment were key zones in regulating terrestrial to aquatic fluxes of OC. 